\title{
BIOGAS PRODUCTION FROM WASTE GLYCEROL WITH INHIBITION FROM CARBOXYLIC ACIDS
}

\author{
V. Beschkov \\ I. Angelov \\ P. Petrova* \\ Institute of Chemical Engineering, *Institute of Microbiology \\ Bulgarian Academy of Sciences, Bulgaria
}

\begin{abstract}
One of the ways for waste glycerol utilization is biogas production. This biogas could be used for partial energy supply of the main biodiesel plant. An important hinder for this application is the accumulation of carboxylic acids leading to strong inhibition of the methanogenesis and shift to production of gas with very low methane content.

The present paper studies the possibility of biogas production from waste glycerol in a multistage anaerobic digester composed by consecutively situated cells separated by baffles. This cascade-type bioreactor enables the separation of zones with different $\mathrm{pH}$ and organic acid content and therefore to achieve flexible operation.

The experiments show stable $\mathrm{pH}$-profile along the bioreactor stages with minimum $\mathrm{pH}$-values in the middle cells. Identification of the intermediate products resulting of the digestion was carried out. A strong correlation between the acid content and the $\mathrm{pH}$-value in each reactor stage was observed.

Microbiological study shows that the microbial profile along the reactor varies with the progress of fermentation. The initial glycerol biodegradation is accomplished by aerobes and facultative anaerobes, whereas the methanogenesis starts after the first stage and it is represented consecutively along the stages by the genera Methanosarcina, Methanobacterium and Methanobrevibacter. Their concentrations are strongly sependent on the $\mathrm{pH}$ values. It was shown that overloading with glycerol leads to gradual decrease of methane in the produced biogas with an un-desired long period of further restoration of the normal reactor functioning.
\end{abstract}

\section{KEYWORDS}

Biogas production; Glycerol utilization; Intermediate inhibition; Multi-stage bioreactor; Microbial profile.

\section{INTRODUCTION}

The present work is associated with the utilization of crude glycerol, a residue from biodiesel production. The amount of this waste product is comparable with the methanol used at the trans-esterification and consists of about $10 \%$ from the produced fuel. The poor quality of this glycerol, containing water, potassium hydroxide and some methanol makes it un-appropriate https://doi.org/10.15626/Eco-Tech.2010.062 
for market purposes even after purification. Additional obstacle for its direct use is the relatively small demand by the pharmaceutical industry, explosives production, etc. That is why different methods for its utilization in production of chemicals (e.g., 1,3-propanediol [1], 2,3-butanediol [2], dihydroxyacetone [3]), some organic acids (lactic [4], propionic [5], succinic [6], etc.) and some polymers [7] have been developed. There are some studies on direct use of glycerol for fuel cells [8].

One attractive alternative for glycerol utilization consists in its direct conversion into biogas, enabling to supply the biodiesel plant with energy. However, problems with the accumulation of organic acids as intermediates and the resulting $\mathrm{pH}$ drop poses an barrier to this application, because of the strong inhibition and even death of the methanogenic bacteria $[9,10]$. It is reported that small amounts of glycerol added to other substrates lead to enhancement of biogas production, but with no real practical applications.

There are different ways to overcome this hinder to biogas production from glycerol. The first one is to maintain the $\mathrm{pH}$ value with alkaline additives, but it will result in undesirable alkalization of the rest wastewater, because of the acids decarboxylation during the biogas production.

Another alternative is to remove the acid intermediates when $\mathrm{pH}$ drops below the optimum value. It will be suitable if these acids are commercially interesting.

The third one is to select an appropriate bioreactor design in order to minimize the inhibitory effect of the acid intermediates.

All these considerations require the experimental study of biogas production from glycerol to establish the admissible load of the bioreactor by substrate, to identify the intermediates and to select an appropriate bioreactor design.

The present paper is a step in this direction.

\section{MATERIALS AND METHODS}

\subsection{Materials and equipment}

Crude glycerol remaining from biodiesel production containing $20 \%$ wt. of water with $\mathrm{pH} \sim 5$ was used. No $\mathrm{pH}$-adjustment was carried out.

A principal sketch of the used bioreactor is shown in Figure 1. It consists of eight rectangular sections with equal volumes separated by stationary baffles with static mixers. The total bioreactor volume was 270 liters. The reactor was initially inoculated by activated sludge with waste stillage from ethanol production as a carbon source. The inoculum and the initial feed were equal for each section. The gas space above the liquid was equal for the whole apparatus. The cultivation was carried out under naturally attained anaerobic conditions, without deliberate purging the bioreactor with inert gas. After full development of the biomass, indicated by the release of combusting biogas the feeding in the first section with crude glycerol started. The excess liquid from each section enters the next one through overflows and below the separating baffles and leaving the apparatus from the end section. The process was carried out in a fed-batch mode. The temperature was maintained at $30-35^{\circ} \mathrm{C}$ by thermostat. Different amounts of crude glycerol (from 0.1 to $0.8 \mathrm{l} /$ day) were added. The amount of the produced gas was periodically measured after collection in a gas-holder under water and the methane content was evaluated by Orsat apparatus. 


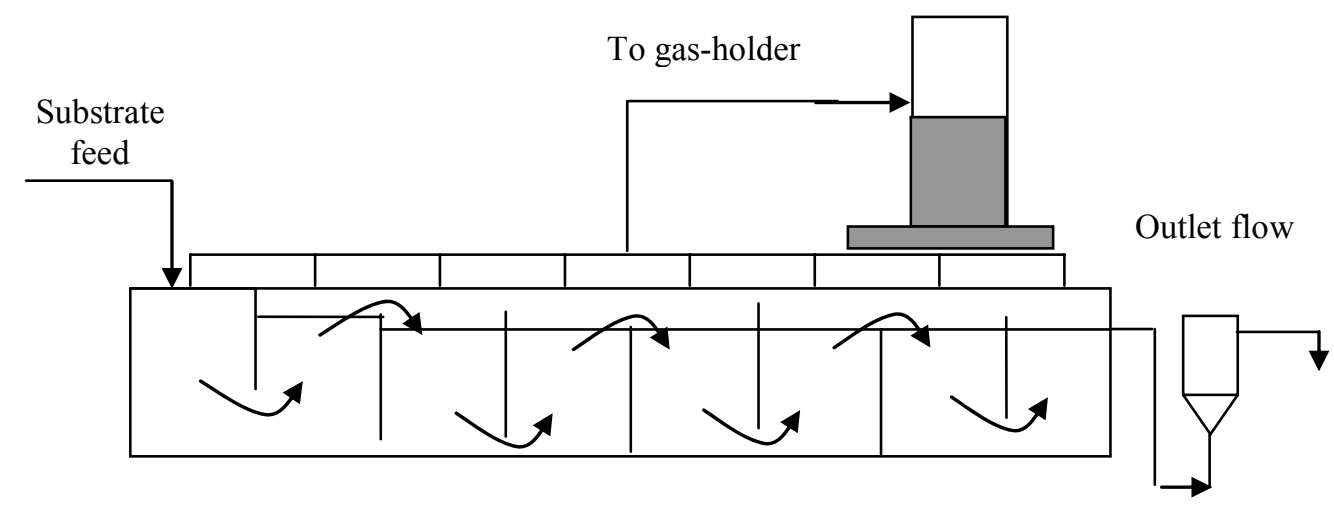

Figure 1. Sketch of the used bioreactor.

Samples from the liquid from each section were taken periodically, filtrated by filters with 0.22 micrometers pore diameter and have been analyzed by HPLC for different intermediate components. The $\mathrm{pH}$ value for each section was measured too.

\subsection{Analyses}

The composition of the liquids in each reactor section was determined qualitatively and quantitatively by HPLC. For this purpose a HPLC chromatograph, Perkin Elmer Series 10, with Bio-Rad column for organic acid analysis (Aminex HPX-87H) was used. The organic acids were determined by Knauer UV-detector at $210 \mathrm{~nm}$, whereas for analyses of alcohols a RI detector was used. Solution of $0.01 \mathrm{~N}$ sulfuric acid was used as a mobile phase at an elution flow rate of $0.6 \mathrm{l} / \mathrm{min}$ at $65^{\circ} \mathrm{C}$. The analyzed intermediates were identified by their retention times compared with added standards.

The $\mathrm{pH}$-values were measured by glass probe coupled to standard $\mathrm{pH}$-meter.

The microbiological analysis of the microbes contained in each section for identification of their genera was accomplished. For this purpose different growing media for aerobes and for anaerobes were used as follows.

For aerobes: meat-peptone agar; Kligler iron agar and chrome coliforme agar;

For anaerobes: Selective thio-lycolate medium with resazurin for anaerobes and ATCC medium for methanogenes, containing $20 \%$ yeast extract, $20 \%$ sodium formate, $25 \%$ sodium acetate, $0.2 \% \mathrm{FeSO}_{4} .7 \mathrm{H}_{2} \mathrm{O}, 0.1 \%$ resazurin and sodium bicarbonate.

The samples from the different reactor sections were grown on these media. The microbe genera were established and their concentrations were estimated semi-quantitatively.

\section{RESULTS AND DISCUSSION}

The selected bioreactor design enables the spatial distribution along the sections of the microbial consortia with specific metabolism and role in the complicated processes of glycerol to biogas conversion. In this manner, different microbial genera are cultivated in the different sections depending on the progress of the glycerol digestion process. This multistage bioreactor operates like plug-flow one facilitating the removal of inhibiting intermediates from the front sections and diluting them in the next ones. That is why this type of bioreactor is very stable to shock overloading with substrate, inhibitors and to different perturbations in the feed and in the temperature regime. 


\subsection{Biogas production}

The time profile in biogas production is shown in Figure 2. It is seen that although the biogas flow rate is high, it decreases at longer duration of the process, reaching zero, or resulting in a non-combustible gas. This effect is explained by the accumulation of acid intermediates demonstrated by the $\mathrm{pH}$-profiles along the bioreactor sections and their variation in time, see Figure 3.

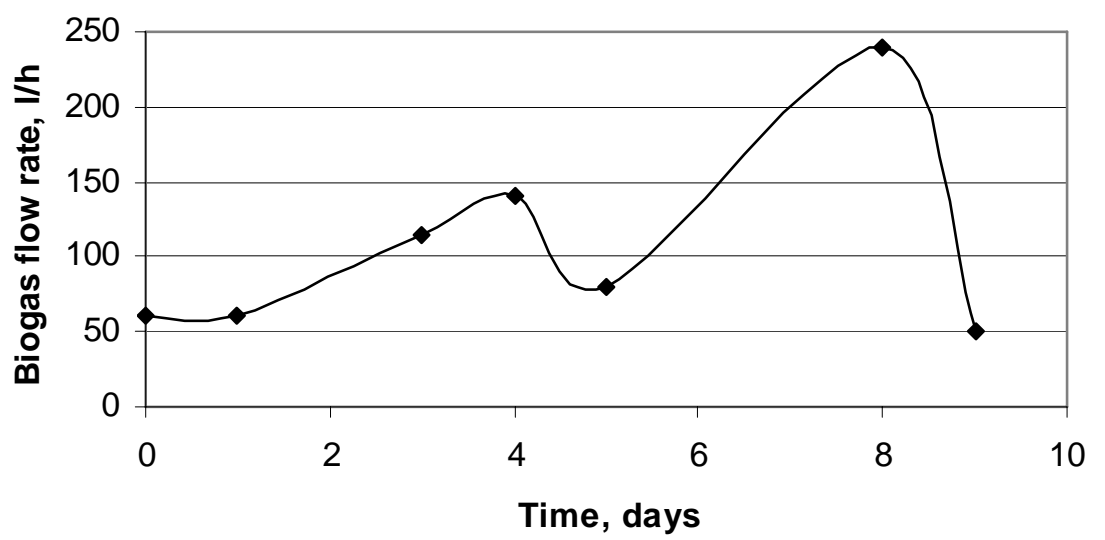

Figure 2. Biogas flow rate at feed of 0.6 l crude glycerol per day.

\subsection{Intermediates profiles and $\mathrm{pH}$ variation}

It is seen that although the $\mathrm{pH}$ variation in the different section, there is a trend for a $\mathrm{pH}$ decrease, due to the formed acids. However, it is evident that $\mathrm{pH}$ values are somewhat lower in the first sections reaching lowest values in Sections 2-4. In the last sections $\mathrm{pH}$ is higher because of the conversion of the organic acids into methane, hydrogen and carbon dioxide, see the consecutive processes, shown in Scheme 1 [11]. It has to be kept in mind however, that the presented scheme is valid for complex substrates, like manure, carbohydrates, where their hydrolysis to simpler compounds is the first step. In the considered case glycerol as rather simple compound is easily converted to different acid products to lower the $\mathrm{pH}$ drastically. There is no well established metabolic pathway for the process of glycerol to methane conversion. However, there are data about the glycerol digestion by the bacteria from

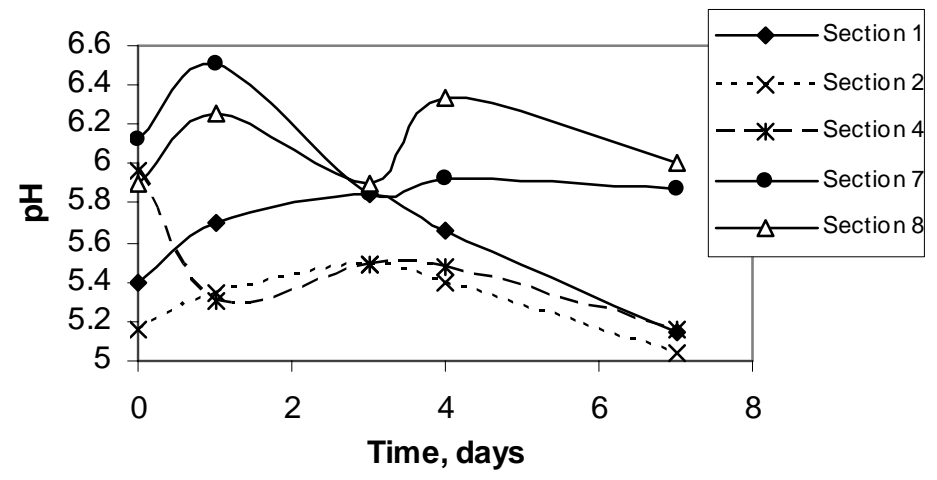

Figure 3. pH variation in time for different reactor sections. 


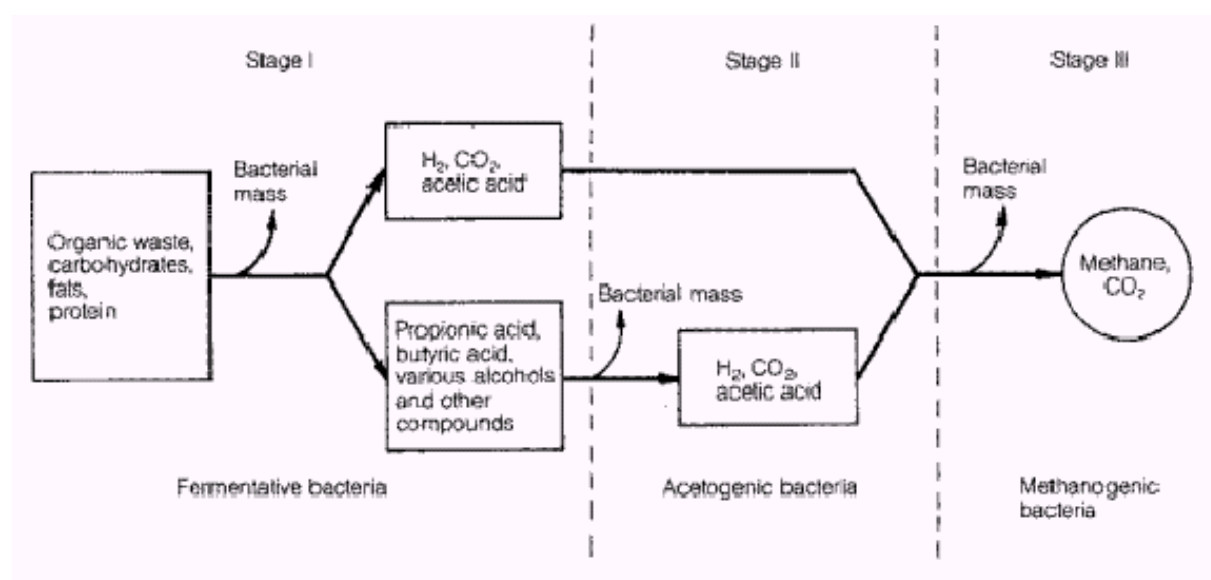

Scheme 1. Pathways for organic matter conversion into methane [11].

the genus Klebsiella following reductive and oxidative metabolic pathways, see Scheme 2 [12].

One can expect that formation of acetic acid and formic acid will lead to methane production in the last step of this scheme. The formation of 2,3-butanediol is a competitive process which may lead to production of this value-added chemical. No propionic acid is involved in this metabolism.

Our attention is drawn mainly on the acid accumulation and their adverse effect on the fermentation. A comparison of the distribution of the organic intermediates along the reactor with the resulting pH-profile is shown in Figure 4.

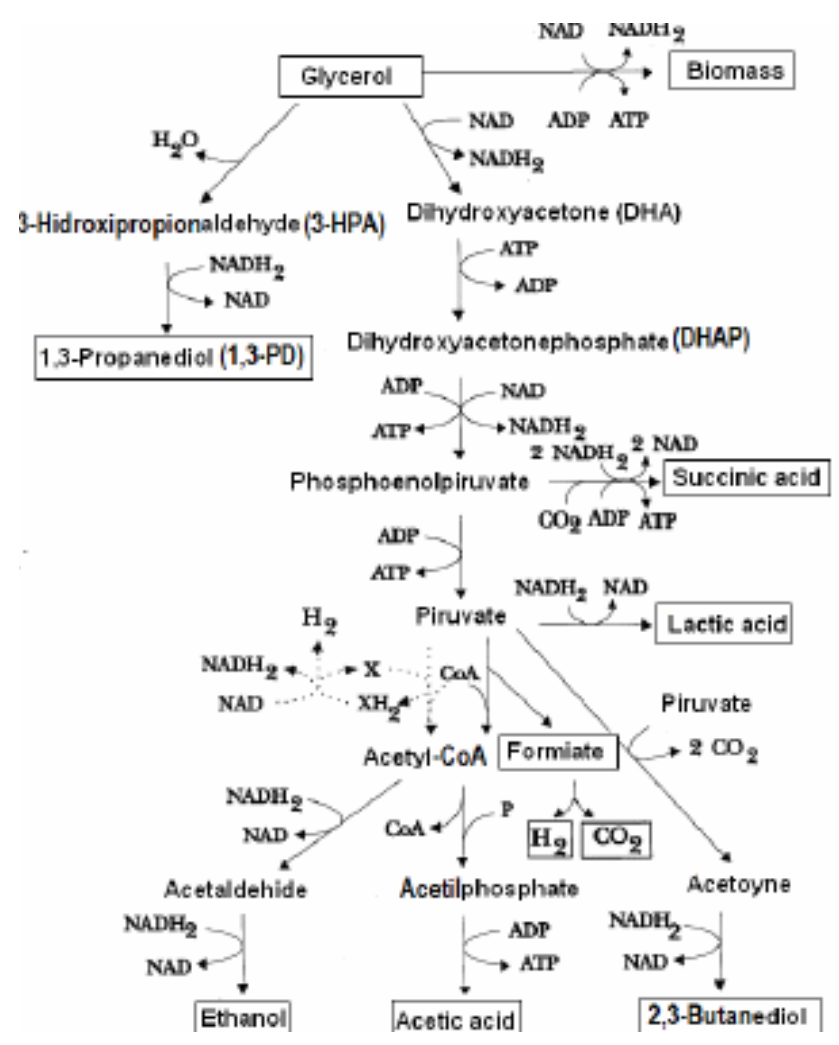

Scheme 2. Anaerobic glycerol metabolism in Klebsiella sp. [12]. 


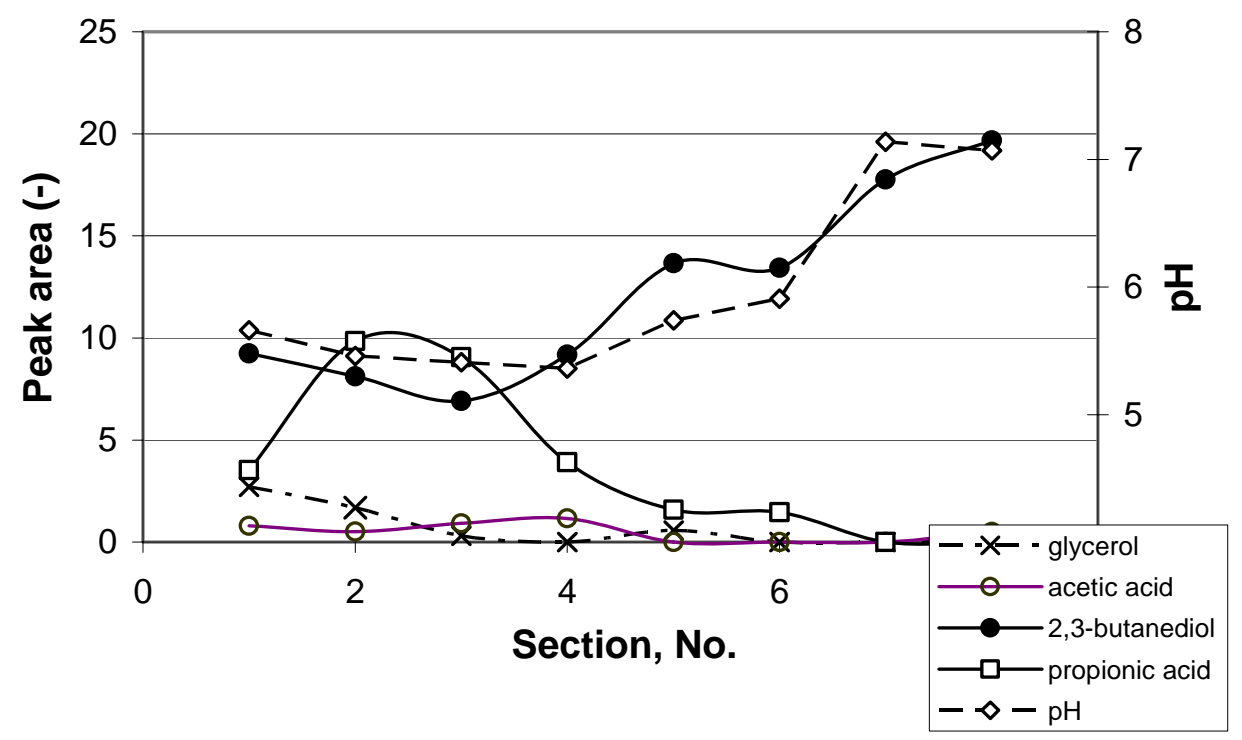

Figure 4. Concentration profiles for intermediates of glycerol metabolism along the reactor sections. Peak areas in HPLC-chromatograms correspond to concentrations.

The level of acetic acid is extremely low, i.e. it is easily converted into methane. There is a maximum in propionic acid concentration corresponding to low $\mathrm{pH}$ values and low 2,3butanediol concentration in Sections 2 and 3. After its degradation in Sections 4-8, pH and 2,3-butanediol concentrations raise. The optimum $\mathrm{pH}$ values are attained in Sections 6-8 where the main amount of methane is produced. Our data show that the multistage design of our bioreactor enables to separate the regions with high and low concentrations of inhibitors, thus enabling the production of biogas and desired metabolites in some section of the reactor. The results of our microbiological analysis support these considerations. The microbial content distribution along the bioreactor sections is shown in Table 1. The presence of propionic acid in our reactors suggests the existence of other microbes except Klebsiella.

Table 1. Microbial profile along the reactor sections

\begin{tabular}{c|c|c|c|c}
\hline Section & \multicolumn{2}{|c|}{$\begin{array}{c}\text { Dominating aerobes, facultative } \\
\text { included } \\
\text { Bacteria count in } 1 \mathrm{ml}\end{array}$} & \multicolumn{2}{c}{$\begin{array}{c}\text { Anaerobes } \\
\text { Bacteria count in } 1 \mathrm{ml}\end{array}$} \\
\hline 1 & $\sim 1 \times 10^{2}$ & Moulds, Bacillus & $\sim 1 \times 10^{1}$ & $?$ \\
\hline 2 & $\sim 1 \times 10^{3}$ & Klebsiella & $\sim 1 \times 10^{5}$ & Methanosarcina \\
\hline 3 & $\sim 1 \times 10^{3}$ & Klebsiella & $\sim 1 \times 10^{5}$ & Methanobacterium \\
\hline 4 & $\sim 2 \times 10^{3}$ & Klebsiella & $\sim 1 \times 10^{6}$ & Methanobacterium \\
\hline 5 & $6-8 \times 10^{2}$ & Klebsiella & $4-5 \times 10^{6}$ & Methanobacterium \\
\hline 6 & $\sim 4 \times 10^{2}$ & Klebsiella & $\sim 2 \times 10^{6}$ & Methanobrevibacter \\
\hline 7 & $1-2 \times 10^{3}$ & Klebsiella & $\sim 1 \times 10^{6}$ & Methanobrevibacter \\
\hline 8 & $\sim 1 \times 10^{2}$ & Bacillus, Klebsiella & $\sim 1 \times 10^{5}$ & Methanobrevibacter \\
\hline
\end{tabular}


The co-existence of Klebsiella and the methanogenes in the reactor suggested that a part of methane is derived from acetate $\left(\mathrm{CH}_{3} \mathrm{COO}^{-}\right)$. Archaea that catabolize this for energy are referred to as acetotrophic or aceticlastic. The Klebsiella bacteria probably convert glycerol into organic acids, including acetic one. Methylotrophic archaea utilize methylated compounds such as methylamines, methanol, and methanethiol as well. The methanogenes from the genera Methanosarcina preferably turn acetic acid into methane after decarboxylation, whereas Methanobacterium and Methanobrevibacter degrade acetic acid into hydrogen and carbon dioxide, yielding methane later. Hydrogen and carbon dioxide are also produced after decomposition of formate by Klebsiella bacteria, as shown in Scheme 2. The results of our microbiological analysis support these considerations.

\section{CONCLUSION}

The main problem in biogas production from glycerol is the accumulation of acid intermediates and the sensitivity of the methanogenic bacteria to acid media. That is why removal of the acid intermediates from the broth is very important for biogas production. This approach may serve for production of value-added products and biogas simultaneously. The chosen reactor configuration facilitates this technique since extraction of intermediates could be deliberately accomplished from a desired reactor section.

Acknowledgement. This study is supported by Grant DTK-36/2009 of the National Fund for Scientific Research of Republic of Bulgaria.

\section{REFERENCES}

[1] Biebl H., Menzel K., Zeng A.P., Deckwer W.D., 1999. Microbial production of 1,3propanediol. Appl. Microbiol. Biotechnol. 52, 289-297.

[2] Biebl H., Zeng A.P., Menzel K., Deckwer W.D., 1998. Glycerol fermentation to 1,3propanediol and 2,3-butanediol by Klebsiella pneumoniae. Appl. Microbiol. Biotechnol. $50,24-29$.

[3] Hu, Z. C., Liu Z.Q., Zheng Y. G., Shen Y.C., 2010. Production of 1,3-dihydroxyacetone from glycerol by Gluconobacter oxydans ZJB09112. J. Microbiol. Biotechnol. 20, 340345.

[4] Biebl, H. Fermentation of glycerol by Clostridium pasteurianum - batch and continuous culture studies,. 2001. J. Ind. Microbiol. Biotechnol. 27, 18-26.

[5] Himmi, E.H. Borie, A., Boussaid, Hassani, A., 2000. Propionic acid fermentation of glycerol and glucose by Propionibacterium acidipropionici and Propionibacterium freudenreichii ssp. shermanii, Appl Microbiol Biotechnol. 53, 435-440.

[6] Pyung Cheon Lee, Woo Gi Lee, Sang Yup Lee, Ho Nam Chang., 2001. Succinic acid production with reduced by-product formation in the fermentation of Anaerobiospirillum succiniciproducens using glycerol as a carbon source, Biotechnol. Bioeng. 72, 41-48.

[7] Ashby, R.D., Wyatt, V.T., Foglia, T.A., Solaiman, D.K.Y., 2009. Industrial products from biodiesel glycerol, in: Biocatalysis and Bioenergy (Ching T. Hou, Jei-Fu Shaw, editors), J. Wiley\&Sons Inc., Hoboken, N.J., pp. 131-149.

[8] Arechederra, R.L, Treu, B.L., Minteer, S.D., 2007. Development of glycerol $/ \mathrm{O}_{2}$ biofuel cell, J. Power Sources, 173, 156-161.

[9] López, J.A.S., Santos, M.A.M., Pérez, A.F.C., Martín, A.M., 2009. Anaerobic digestion of glycerol derived from biodiesel manufacturing, Bioresource Technol., 100, 5609-5615.

[10] Kolesárová, N., Hutňan,M., Špalková,V., Lazor, M., 2010. Biodiesel by-products as potential substrates for biogas production. Proc. 37 th International Conference of Slovak 
Society of Chemical Engineering, Tatranské Matliare, Slovakia, May 24 -28, pp. 11261139.

[11]. Maerkl, H. 1980. Mikrobielle Methangewinnung, in: Fortschritte der Verfahrenstechnik. 1980, VDI-Verlag: Düsseldorf D. p. 509 - 525.

[12]. Zhang Y., Huang Zh., Du, Li Y., Cao Z., 2009. Introduction of an NADH regeneration system into Klebsiella oxytoca leads to an enhanced oxidative and reductive metabolism of glycerol, Metabolic Engineering, 11, 101-106. 